\title{
Composición florística del bosque ripario en la microcuenca La Laguneta, municipio de Pueblo Nuevo, Estelí
}

\section{A floristic composition of the riparian forest in La Laguneta's microwatershed, Pueblo Nuevo municipality, Estelí}

\author{
Emilio Pérez-Castellón ${ }^{1}$, Glenda Bonilla-Zúñiga', Elvin Santiago Díaz-Rivera² \\ ${ }^{1}$ Departamento de Manejo de Cuencas, Universidad Nacional Agraria, emiliopc@ci.una.edu.ni, emiliopc21@hotmail.com, \\ gbonilla@una.edu.ni, ${ }^{2}$ Graduado UNA, elvinrivera30@yahoo.es
}

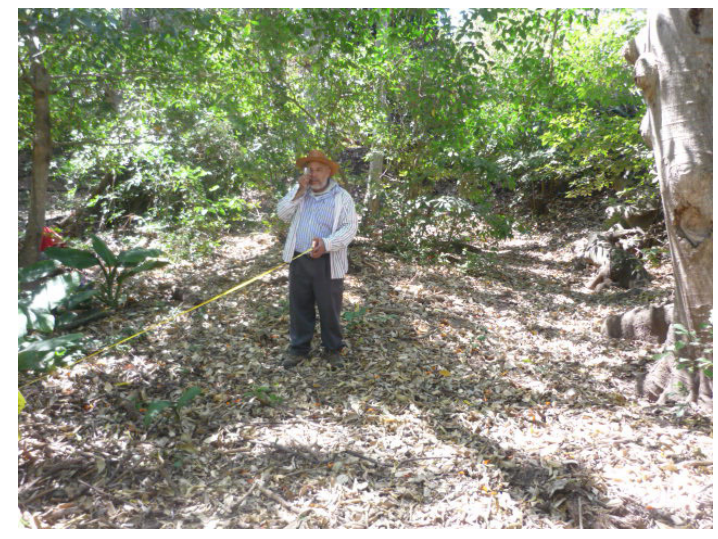

\section{RESUMEN}

Este estudio se deriva del proyecto UNA/CARE/MARENA-PIMCHAS y se realizó en la microcuenca La Laguneta, sitio ubicado en el municipio de Pueblo Nuevo, departamento de Estelí El propósito fue evaluar la composición florística, del bosque ripario. La investigación se realizó en tres etapas, la primera comprende el reconocimiento del área de estudio, la segunda, el inventario forestal en el bosque ripario y la tercera corresponde el análisis de la información recopilada. El levantamiento de la información se realizó a través de un inventario sistemático en parcelas de $10 \mathrm{~m}^{2}$ con un transecto paralelo a la ribera del rio. El total de parcelas fueron 23 y en cada una se seleccionaron los árboles mayores a los $10 \mathrm{~cm}$ de diámetro a la altura del pecho y se ubicó una subparcela de un $\mathrm{m}^{2}$ para inventariar todas las plantas menores a los $10 \mathrm{~cm}$ de diámetro. Se calcularon los índices de diversidad de Shannon y Simpson para la parte alta, media y baja de la microcuenca. El estudio demuestra mayor diversidad en la parte alta y menor en la parte baja del sitio. En el inventario de árboles mayores de $10 \mathrm{~cm}$ de diámetro se registraron 31 especies representadas en 21 familias botánicas, sobresaliendo la Mimosácea (seis), para la regeneración natural, se contabilizaron 12 especies representadas en ocho familias botánicas. En total se encontraron 300 árboles por hectárea, de los cuales la mayoría pertenecen a las clases diamétrica $10-19.9 \mathrm{~cm}$, que son las más representativas y $27.0915 \mathrm{~m}^{2} \mathrm{ha}^{-1}$ de área basal, registrándose en mayor concentración en las categorías de 50-59.9 y >60 cm. Existe deterioro ambiental por deforestación en la parte baja debido a la actividad agrícola y menos afectación por la misma actividad en la parte alta.
The study was conducted in the watershed La Laguneta, site located in the municipality of Pueblo Nuevo, Esteli department, in order to assess the species composition of riparian forest. The research was conducted in three stages: the first involves the recognition of the study area, the second forest inventory in the riparian forest and the third analysis of the collected information. The collection of information is done through a systematic inventory on plots of $10 \mathrm{~m} 2 \mathrm{con}$ a transept parallel to the banks of the river. The total number of plots were 23, where each took all trees greater than $10 \mathrm{~cm} \mathrm{dbh}$ and within this stood a $1 \mathrm{~m} 2$ subplot to inventory all the plants under $10 \mathrm{~cm}$ in diameter. We calculated the Shannon diversity index and Simpson to the top, middle and lower watershed. he study shows greater diversity in the upper and lower in the bottom of the site. In the inventory of trees over $10 \mathrm{~cm}$ were found 31 species in 21 plant families represented projecting the Mimosacea (6), for natural regeneration found 12 species in 8 plant families represented. A total of 300 trees per hectare found of which most belong to 10-19.9 $\mathrm{cm}$ diameter classes that are most representative and $27.0915 \mathrm{~m}^{2} / \mathrm{ha}$ basal area which is found in greater concentration in the categories of 50-59.9 and $>60 \mathrm{~cm}$. There is environmental degradation due to deforestation in the lower due to agricultural activity. In the same study found that the top is the least affected by agricultural activities. This study was one of several Bachelor theses, funded by the project UNA/CARE/MARENA-PIMCHAS. 
$\mathrm{E}$ n Nicaragua al igual que otros países centroamericanos las actividades productivas principales son la agricultura y la ganadería extensiva, por lo tanto, el recurso forestal y el uso de la tierra están sometidos a una dinámica que paulatinamente cambian su extensión y estructura, esto debido al avance de la frontera agrícola que provoca degradación y deforestación (Filomeno, 1996).

Las vegetaciones riparias varían ampliamente en sus características físicas, las cuales se expresan vívidamente a través de un gran número de estrategias de historial natural y patrones de sucesión. Consecuentemente estas áreas se encuentran entre los ecosistemas ecológicos más complejos de la biósfera y también de los más importantes para mantener la vitalidad del paisaje y sus ríos dentro de las cuencas hidrográficas (Naiman et al., 2000).

Los bosques riparios de las eco-regiones del mundo son florísticas y estructuralmente los más diversos, y su conservación debería ser un componente integral para las estrategias de manejo de cuencas hidrográficas (Naiman et al., 2000).

El papel que juega el bosque ripario es retardar y reducir la escorrentía superficial, utilizando el exceso de nutrientes, atrapando los sedimentos y otros contaminantes que se desprenden de los suelos descubiertos o suelos de cultivos, además protegen los cuerpos de agua y aumentan la infiltración en las áreas de inundación por acción de las raíces de las plantas que crecen en estas aéreas (Blinn y kilgore, 2001).

Las zonas riparias proveen de hábitat, así como también una vía para el desplazamiento de la vida silvestre de un parche de vegetación a otro, tanto en ambientes fragmentados como continuo. Entre mayor sea la conectividad entre los parches y hábitats, los animales encontraran más fácil el desplasmiento entre sitios. Esto ayuda a mantener las poblaciones de vida silvestre en bosques y parches de áreas arboladas (Naiman et al., 2000).

Existen muy pocos trabajos realizados en los bosques riparianos o de galería -como son comúnmente denominados- en el ámbito de su composición florística, considerando desde la parte alta hasta la parte baja de las microcuencas, como el caso de este trabajo. Normalmente hay estudios de composición florística e inventarios solo en fracciones de las cuencas y no en todo su trayecto, desde sus nacimientos hasta sus desembocaduras.

El proyecto UNA/CARE/MARENA-PIMCHAS persigue contribuir al mejoramiento de la calidad de vida y al bienestar económico de cinco microcuencas de Estelí y Jinotega, ya que su estado se encuentra en deterioro.

El presente trabajo está enfocado en evaluar la composición florística del bosque ripario en la microcuenca La Laguneta, donde el proyecto UNA/CARE/MARENA-PIMCHAS desarrolló inversiones y acciones con enfoque de manejo de cuenca.

\section{MATERIALES Y MÉTODOS}

Ubicación geográfica. La microcuenca La Laguneta se encuentra ubicada a cuatro $\mathrm{km}$ al sur del municipio de Pueblo Nuevo en departamento de Estelí y a $216 \mathrm{~km}$ de la capital de Nicaragua, Managua. Se ubica entre las coordenadas $13^{\circ} 22^{\prime} 60$ de latitud norte y $86^{\circ} 28^{\prime} 0$ longitud oeste.
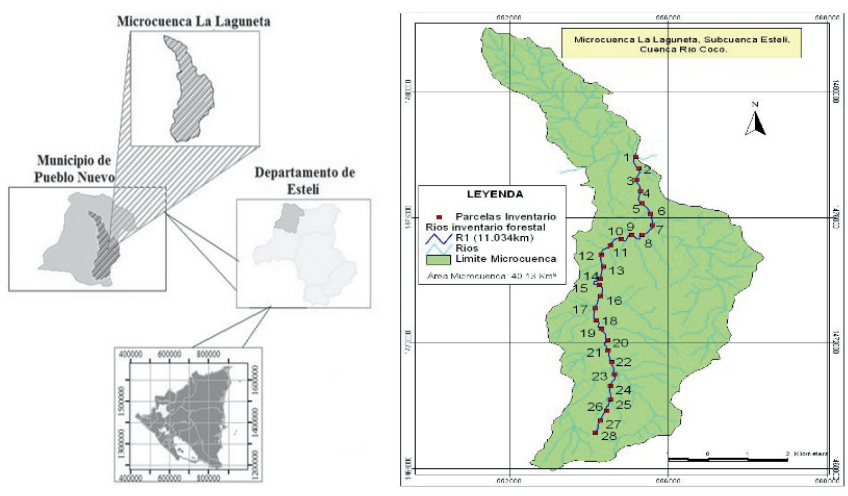

Figura 1. Ubicación geográfica de la microcuenca (a) y distribución de parcelas de muestreo (b).

Clima. El clima de la microcuenca es del tipo monzónico de tierras altas, caracterizado como subtropical seco pero bastante fresco.

Fisiografía. El área está situada en la provincia fisiográfica "Tierras Altas del Interior" caracterizada por ser moderadamente escarpada o muy extremadamente escarpada (Rodríguez et al., 1995).

Recursos forestales. Las áreas de vocación o aptitud forestal, han sido deforestadas y convertidas en pastizales, dejando únicamente las especies de poco valor forestal (Rodríguez et al., 1995).

Diseño y aplicación del inventario en el bosque ripario. El diseño del inventario consistió en un muestreo sistemático, tomando como línea base la ribera del río principal. Se delimitaron 23 parcelas de $100 \mathrm{~m}^{2}(10 \mathrm{~m}$ X $10 \mathrm{~m})$ equivalente a 0.01 ha cada una y separadas a 400 metros una con respecto a otra.

Levantamiento de información. Se calculó la intensidad de muestreo (\%) para la vegetación mayor a $10 \mathrm{~cm}$ de DAP y para la regeneración natural. La intensidad de muestreo se realizó sobre la base de la relación del tamaño de la muestra (Tm) y el área total (At) expresado en hectárea. Esta proporción se transformó a porcentaje al multiplicarse por 100, dando como resultado $0.69 \%$,-ya que la longitud del rio es de 9430 metros i??.-La metologia utilizada es la propuesta por el CATIE (2001). 
a) $I M \%=(\mathrm{Tm} / \mathrm{At}) \times 100$

Donde:

$\mathrm{IM}=$ Intensidad de muestreo

$\mathrm{Tm}=$ Tamaño de la muestra (ha)

At $=$ Área total (ha)

\section{b) $\mathrm{Tm}=\mathrm{Tp} \times \mathrm{Np}$}

Donde:

$\mathrm{Tm}=$ Tamaño de la muestra (ha)

$\mathrm{Tp}=$ Tamaño de la parcela (ha)

$\mathrm{Np}=$ Número de parcela

Variables dasométricas

Diámetro a la altura del pecho (DAP). Se utilizó una cinta diamétrica. El Área basal es una aproximación del área de la sección transversal de un árbol. Se deduce de la ecuación del círculo, y se calcula por la siguiente fórmula (Prodan et al., 1997).

$\mathrm{AB}=0.7854 \mathrm{DAP}^{2}$

Composición florística. Se registró información sobre la vegetación con diámetro a la altura del pecho (DAP) mayor de $10 \mathrm{~cm}$, así como la vegetación correspondiente a la regeneración natural o vegetación menor a los $10 \mathrm{~cm}$ de DAP.

\section{Variables silviculturales}

Iluminación. Tiene que ver con la influencia de la luz solar de los diferentes estratos del bosque donde se encuentran ubicados los individuos (Carrera, 1994).

Infestación por lianas. La infestación por especies de lianas y trepadoras tiene serios efectos en el incremento, forma de los árboles, sobrevivencia y producción futura de madera (Synnott, 1991).

Calidad de fuste. Se tomó de acuerdo al criterio de observación (Hutchinson, 1993) clasificándola en el rango de buena (1), regular (2) y mala (3). Además se consideró la vigorosidad, determinada a través de la verificación del estado físico que presentan los árboles al momento de su observación, y representa el grado de adaptación del árbol al medio en que se desarrolla, la que puede ser afectada por causa natural o por efectos de agentes externos como incendios. La vigorosidad se determinó según la clasificación de Hutchinson (1993), catalogada como vigoroso (1), media (2) y pobre (3).

Índices de biodiversidad. Son herramientas que nos permiten tener una perspectiva de la situación de la comunidad, con el fin de realizar monitoreo ambientales y tomar decisiones de conservación y manejo (Spellerberg, 1991).
Índice de Shannon-Wiener. Es uno de los índices más utilizados para determinar la diversidad de especies de un determinado hábitat. Es utilizado para expresar la uniformidad de los valores de importancia a través de todas las especies de la muestra (Moreno, 2001).

Índice de Simpson. Es otro método utilizado comúnmente para determinar la diversidad de una comunidad (Mostacero y Fredericksen, 2002).

Índice de valor de importancia (IVI). Este índice resulta de la suma de los valores relativos de la abundancia, la frecuencia y la dominancia (Lamprecht, 1962).

\section{RESULTADOS Y DISCUSIÓN}

Composición florística. Se registraron 69 individuos con DAP mayor de $10 \mathrm{~cm}$ que pertenecen a 31 especies arbóreas distribuidas en 21 familias botánicas, de las cuáles las más representativas son Fabaceae con cinco árboles, Mimosaceae con tres árboles, Boraginaceae con dos árboles y Anacardiaceae con dos. De las 23 parcelas establecidas, se encontraron seis sin vegetación $(26.09 \%)$ y 17 parcelas con vegetación equivalente a $73.93 \%$.

En cuanto a la regeneración natural o vegetación menor a los $10 \mathrm{~cm}$ de DAP, se registraron 12 especies representadas por ocho familias botánicas, siendo la más representativa la Mimosaceae. La densidad poblacional fue de 6957 plantas por hectárea.

Distribución diamétrica de las especies mayores a $10 \mathrm{~cm}$ DAP. La distribución diamétrica del total de individuos tuvo un patrón de "J" invertida, lo que indica que se registró mayor número de individuos en las categorías menores y menor número en las categorías mayores. El mayor número de individuos se clasifica en la clase diamétrica de 10-19.9 cm representado por 34 individuos, equivalente a 148 árboles por hectárea, seguido por la segunda clase diametrica $20-29.9 \mathrm{~cm}$ (17 individuos) que equivalente a 74 árboles por hectárea. Las cuatro categorías restantes obtuvieron un número de individuos menor en comparación a las categorías anteriores y no sobrepasan los 30 árboles por hectárea (figura 2).

Área basal total. Mayor área basal se registró en la clase diamétrica mayor de $60 \mathrm{~cm}$ de DAP con $3.0414 \mathrm{~m}^{2}$, mientras que las clases diamétricas menores, no sobrepasan un área basal de un $\mathrm{m}^{2}$ ya que son árboles de diámetros que varían entre 10 y $59.9 \mathrm{~cm}$. En cuanto al área basal por hectárea se encontró que la clase diamétrica mayor de 60 centímetros tiene el mayor valor con $13.25 \mathrm{~m}^{2} \mathrm{ha}^{-1}$. En total el área basal en el área muestreada es de $27.0915 \mathrm{~m}^{2} \mathrm{ha}^{-1}$. 


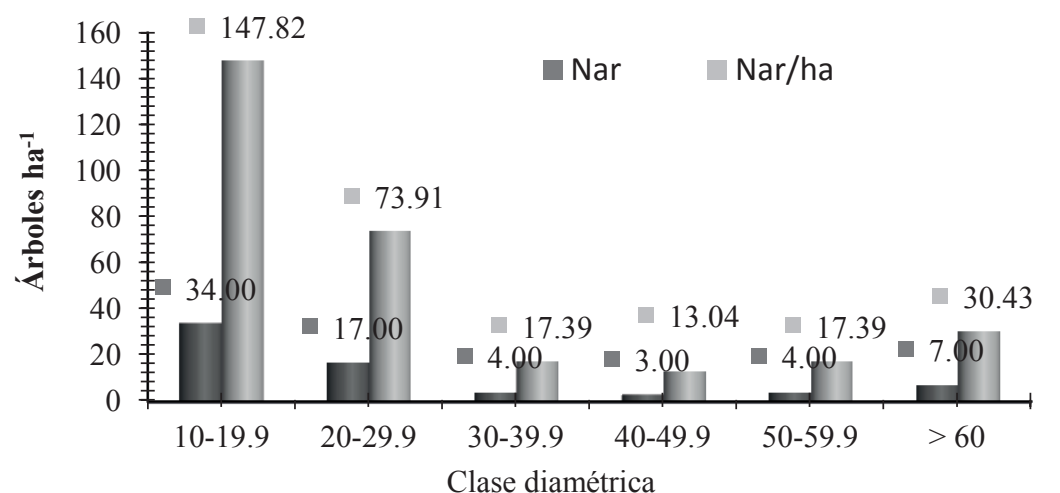

debido al cambio de uso de la tierra ya que los productores buscan áreas con menor pendiente para cultivar y establecer áreas de pasto.

Esto indica que existe mayor presencia de regeneración natural en la parte alta y media de la microcuenca, debido a que en la parte baja de existe mayor concentración de los asentamientos humanos y mayor población, además es un área donde las actividades agrícolas son más frecuentes estableciéndose áreas de granos básicos y ganaderas.
Figura 2. Distribución de árboles por hectárea según clase diamétrica.

\section{Variables silviculturales de las especies mayores de $10 \mathrm{~cm}$ de DAP}

Calidad de fuste. Se registró mayor predominancia de los árboles dentro de la categoría 2 (fuste regular) con 27 árboles $(39.13 \%)$ los que presentan cierto nivel de curvatura, seguido de la categoría 3 (fuste malo) con 23 árboles (33.3\%) con curvaturas bien marcada; por último, en la categoría 1 (fuste bueno) se registran 19 árboles (33.33\%) considerados como árboles sin presencia de defectos en el fuste.

Infestación por lianas. En el inventario se encontró que 55 árboles (79.71\%), no presentan lianas (categoría 1), 1.55\% (1 árbol) presentó solo lianas en el fuste (categoría 2), el 5.80\% (4 árboles) presentaron lianas en el fuste y en la copa pero no afectan el crecimiento (categoría 3) y $13.04 \%$ (9 árboles) presentaron lianas en la copa y en el fuste que si afectan el crecimiento (categoría 4).

Iluminación. El bosque ribereño de la microcuenca La Laguneta presenta un relieve montañoso que se ubica en la posición este-oeste y que a pesar de esto, la luz solar penetra fácilmente en el bosque, no afectando el crecimiento de las especies en todos los estratos del bosque.

Vigor. De acuerdo a la vigorosidad se registró que el 55\% (38 árboles) presentan buen vigor, el 37.68\% (26 árboles) de vigor medio y $7.25 \%$ (cinco árboles) con vigor pobre.

\section{Análisis de diversidad}

Comparación de la riqueza y abundancia. Tanto para árboles mayores y menores de $10 \mathrm{~cm}$ de DAP, la mayor riqueza y abundancia de especies se encuentra en la parte alta de la microcuenca, seguida de la parte media. En la parte baja es donde existe menor riqueza y abundancia de especies
Índice de diversidad Shannon-Wiener y Simpson. Según Shannon-Wiener para las especies mayores de 10 centímetros de diámetro, la diversidad aumenta de la parte baja hacia la parte alta (cuadro 1), lo que debe a que existe una mayor intervención por el hombre y extrae ciertas especies para fines energéticos o para cubrir otras necesidades (madera para postes y construcciones rústicas).

En cuanto a la regeneración natural también existe mayor diversidad en la parte alta, debido a que existe mayor diversidad de especies mayor a los $10 \mathrm{~cm}$ de DAP (árboles frutales) y mayor concentración de la fauna silvestre, principalmente aves, que intervienen en la polinización, contribuyendo con una mayor regeneración. Según el índice de Simpson la diversidad se comportó de igual manera al índice de Shannon-Wiener. Ambos índices indican mayor diversidad en la parte alta.

Cuadro 1. Índice de diversidad según Shannon-Wiener y Simpson según parte alta, media y baja

\begin{tabular}{lcccccc}
\hline \multirow{2}{*}{ Índice } & \multicolumn{3}{c}{ Vegetación $>10 \mathrm{~cm}$ de Dap } & \multicolumn{3}{c}{ Vegetación $<10 \mathrm{~cm}$ Dap } \\
\cline { 2 - 6 } & Parte alta & Parte media & Parte baja & Parte alta & Parte media & Parte baja \\
\hline Shannon-Wiener & 2.887 & 2.281 & 1.977 & 1.561 & 1.242 & 1.386 \\
Simpson & 0.060 & 0.120 & 0.170 & 0.220 & 0.330 & 0.250 \\
\hline
\end{tabular}

Indice de valor de importancia. Las especies de mayor densidad en la microcuenca fueron: Alvaradoa amorphoides Liebm. (caratillo) y Acacia pennatula (Cham. \& Schltdl.) Benth. (carbón) con 34.78 árboles por hectárea respectivamente seguido por el Salix humboldtiana Willd. (sauce) con 21.74 árboles por hectárea. Las restantes especies se encuentran en densidades que varian entre 4.35 a 17.39 árboles por hectárea.

Las especies con mayor importancia ecológica en el bosque fueron: Jacaratia dolichaula (Donn.Smith) R.E.Woodson (papalón) con 27.42 seguido por el Acacia pennatula (Cham. \& Schltdl.) Benth. (Carbón) con 22.30 y Alvaradoa amorphoides Liebm. (Caratillo) con 21.79. Las especie de menor importancia ecológica corresponden a Spondias purpurea L. (jocote) y Cordia alliodora (Ruiz \& Pavón) Oken (laurel negro) con 3.59, Pachira quinata (Jacq.)W.S. Alverson (pochote) 3.58 y Diospyros nicaraguensis Standl. (bum bum) con 3.56 . 


\section{CONCLUSIONES}

La composición florística de la microcuenca la Laguneta se representa en 31 especies y 21 familias botánicas, predominando las familias fabácea, mimosácea, boraginácea y anacardiácea, observándose mayor composición florística en la parte alta y mayor área basal en las categorías mayores de $60 \mathrm{~cm}$.

El bosque ripario recibe en su mayoría iluminación vertical y lateral plena. Adicionalmente, la poca presencia de lianas indica intervención del bosque provocada por pobladores de la zona, predominando árboles con fuste regular debido a la topografía que presenta el sitio. Finalmente la buena vigorosidad de los arboles refleja buenas condiciones ambientales para el desarrollo de los mismos.
La alta diversidad encontrada en la parte alta de la microcuenca con árboles mayores a $10 \mathrm{~cm}$ de DAP, y mayor diversidad para árboles menores a $10 \mathrm{~cm}$ de DAP en la parte baja de la microcuenca, explica la influencia de factores ambientales como el desarrollo de los suelos, la ocurrencia de deslaves, o el afloramiento rocoso y la dificultad para entrar por parte de los comunitarios al bosque. La poca diversidad encontrada en la parte baja está asociada a la ocurrencia de deslaves y al afloramiento rocoso del área.

Las especies con mayor importancia eecológica corresponden a Jacaratia dolichaula (Donn.Smith) R.E.Woodson, Acacia pennatula (Cham. \& Schltdl.) Benth y Alvaradoa amorphoides Liebm. Especies adaptadas a las condiciones ambientales y con mecanismos fáciles de dispersión de sus semillas.

\section{REFERENCIAS BIBLIOGRÁFICAS}

Blinn, R; Kilgore, A. 2001, Prácticas de gestión ribereña, gestión de silvicultura. Universidad de Minesota, US. p. 11-17

Carrera, F. 1994. Guía para la planificación de inventarios forestales en la zona de usos múltiples de la reserva de la biosfera Maya Peten, Guatemala. 40 p.

CATIE (Centro Agronómico de Investigación y Enseñanza, CR). 2001. Silvicultura de bosque latifoliado húmedo con énfasis en América Central Ed. BLouman; D Quiroz; M, Nilson Turrialba, CR. Serie técnica, manual técnico No 46.265 p.

Filomeno, S. 1996. Dinámica del sector forestal en Nicaragua 1960-1965 (Lineamiento para un desarrollo sustentable) editorial INIES, Managua, NI. 212 p.

Hutchinson, D. 1993. Puntos de partida y muestreo, diagnostico para la silvicultura de bosques naturales del tropico humedo. Colección de la silvicultura y manejo de bosques naturales $\mathrm{N}^{\circ} 7.15 \mathrm{p}$.

INETER (Instituti Nicaragüense de Estudios Territoriales). 1995. Fotografias aereas (21) escala 1:25000, año 1998.

Lamprecht, H. 1962. Ensayo sobre unos métodos para el análisis estructural de los bosques tropicales. Acta científica venezolana, Universidad de los Andes, Mérida, Venezuela

Moreno, C. 2001. Métodos para medir la biodiversidad. Vol. 1. M\&T-Manuale y Tesis SEA. Zaragoza - España. 84 p.

Mostacero, B; Fredericksen, S. 2000. Manual de métodos básicos de muestreo y análisis en ecología vegetal. Proyecto de manejo forestal sostenible (BOLFOR). Santa Cruz de la Sierra. Bolivia. 87 p.

Prodan, M; Peter, R; Cox, F; Real, P. 1997. Mensura forestal ED.IICA/GTZ San José CR. IICA. Serie de investigación y educación en desarrollo sostenible $586 \mathrm{p}$.

Naiman, R.; Bilby, R.; Bisson, P. 2000. Ecología ribereña y manejo del bosque lluvioso en la costa del Pacífico BioScience 50 (11). 996-1011 p.

Rodríguez, I; Martínez, C; Acuña, E. 1995. Estudio de reconocimiento y caracterización de los recursos hídricos, edáficos y forestales del municipio de Pueblo Nuevo, Estelí-Nicaragua; edición preliminar. 77 p.

Spellerberg, I. 1991. Monitoring ecological change. Cambridge University Press, UK. 334 p.

Synnott, T. 1991. Manual de procedimiento de parcelas permanentes para bosque húmedo tropical, Instituto Tecnológico de Costa Rica. Serie de apoyo académico No 12.103 p. 